\title{
Real-time all-optical OFDM transmission system based on time-domain optical fourier transformation
}

Guan, Pengyu; Kong, Deming; Røge, Kasper Meldgaard; Mulvad, Hans Christian Hansen; Galili, Michael; Oxenløwe, Leif Katsuo

\section{Published in:}

Proceedings of 2014 Optical Fiber Communications Conference and Exhibition

Link to article, DOI:

10.1364/OFC.2014.W4F.1

Publication date:

2014

Document Version

Peer reviewed version

Link back to DTU Orbit

Citation (APA):

Guan, P., Kong, D., Røge, K. M., Mulvad, H. C. H., Galili, M., \& Oxenløwe, L. K. (2014). Real-time all-optical OFDM transmission system based on time-domain optical fourier transformation. In Proceedings of 2014 Optical Fiber Communications Conference and Exhibition (pp. 1-3). [W4F] IEEE.

https://doi.org/10.1364/OFC.2014.W4F.1

\section{General rights}

Copyright and moral rights for the publications made accessible in the public portal are retained by the authors and/or other copyright owners and it is a condition of accessing publications that users recognise and abide by the legal requirements associated with these rights.

- Users may download and print one copy of any publication from the public portal for the purpose of private study or research.

- You may not further distribute the material or use it for any profit-making activity or commercial gain

- You may freely distribute the URL identifying the publication in the public portal 


\title{
Real-Time All-Optical OFDM Transmission System Based on Time-Domain Optical Fourier Transformation
}

\author{
P. Guan, D. Kong*, K. M. Røge, H. C. H. Mulvad, M. Galili, L. K. Oxenløwe \\ DTU Fotonik, Technical University of Denmark, Ørsteds Plads, 343, Kgs. Lyngby, 2800, Denmark \\ * State Key Laboratory of Information Photonics and Optical Communications, BUPT, Beijing, 100876, China \\ pengu@fotonik.dtu.dk
}

\begin{abstract}
We propose a novel simple all-optical OFDM transmission system based on timedomain OFT using time-lenses. A real-time $160 \mathrm{Gbit} / \mathrm{s}$ DPSK OFDM transmission with 16 decorrelated data subcarriers is successfully demonstrated over $100 \mathrm{~km}$.

OCIS codes: (060.4080) Modulation; (060.4230) multiplexing; (070.7145) ultrafast processing.
\end{abstract}

\section{Introduction}

Optical orthogonal frequency-division multiplexing (OFDM) has received much attention due to its high spectral efficiency [1]. All-optical OFDM performs subcarrier multiplexing and demultiplexing in the optical domain, thereby circumventing speed limitations of electronic IFFT/FFT and digital/analog conversions, and has enabled Tbit/s capacity OFDM super-channels [2,3]. Most proposals for AO-OFDM transmitters use rectangular-like electrical modulation to generate the OFDM subcarriers from individual comb-lines, and then combine them using a coupler [1,2]. However, such transmitters are limited by the electronic bandwidth in realizing the fast symbol transitions of OFDM subcarriers. To avoid this limitation, the OFDM subcarriers can also be generated from datamodulated optical pulses using optical inverse Fourier transforms (OIFT) based on cascaded delay interferometers [4], arrayed waveguide grating routers [5], or wavelength selective switches (WSS) [3]. However, the complexity of these OIFT schemes generally increases with the number of independent data-subcarriers that must be generated.

In this paper, we propose a novel all-optical OFDM transmission system based on time-domain optical Fourier transformation (OFT) [6]. The proposed scheme is essentially simple, and the implementation complexity does not increase with the number of subcarriers, as opposed to the OIFT schemes [3-5]. In the transmitter, all the OFDM subcarriers are simultaneously generated in real-time from an OTDM signal by an OFT in combination with rectangular pulse shaping. This is equivalent to an optical time lens system with a temporal aperture, performing an exchange between temporal and spectral envelopes of the signal within the aperture. In the receiver, the OFDM subcarriers are simultaneously frequency-to-time converted by another OFT [7], followed by individual detection using time-domain optical sampling. We successfully demonstrate a 16×10 Gbit/s DPSK-OFDM signal with $0.8 \mathrm{symbol} / \mathrm{s} / \mathrm{Hz}$ spectral efficiency, followed by $100-\mathrm{km}$ transmission and detection with error-free performance.

\section{Principle}

The principle of the proposed all-optical OFDM transmission system is shown in Fig. 1. In the transmitter, the OFDM signal is generated by first converting an OTDM signal to a WDM signal by OFT, and then shaping the WDM channels into OFDM subcarriers using a temporal pulse shaper (aperture). The OTDM signal has a flat-top spectrum, and the OTDM tributaries have a pulse width of $\delta t_{0}$ and temporal spacing $\Delta T_{I}(\mathbf{A})$. In principle, the OTDM signal can be obtained from a single pulse source as shown in Fig. 1 (mode-locked laser - MLL). The OFT is based on a dispersion medium with second order dispersion $D_{1}=\beta_{2} L$, followed by a quadratic phase-modulation stage with chirp rate $K_{I}=1 / D_{I}$ (a D-K configuration). The OFT performs time-to-frequency conversion from OTDM to WDM, but also frequency-to-time conversion of the flat-top OTDM spectrum into the time-domain. In general, such a complete OFT would require an additional phase-modulation stage with chirp rate $K_{l}$ before the

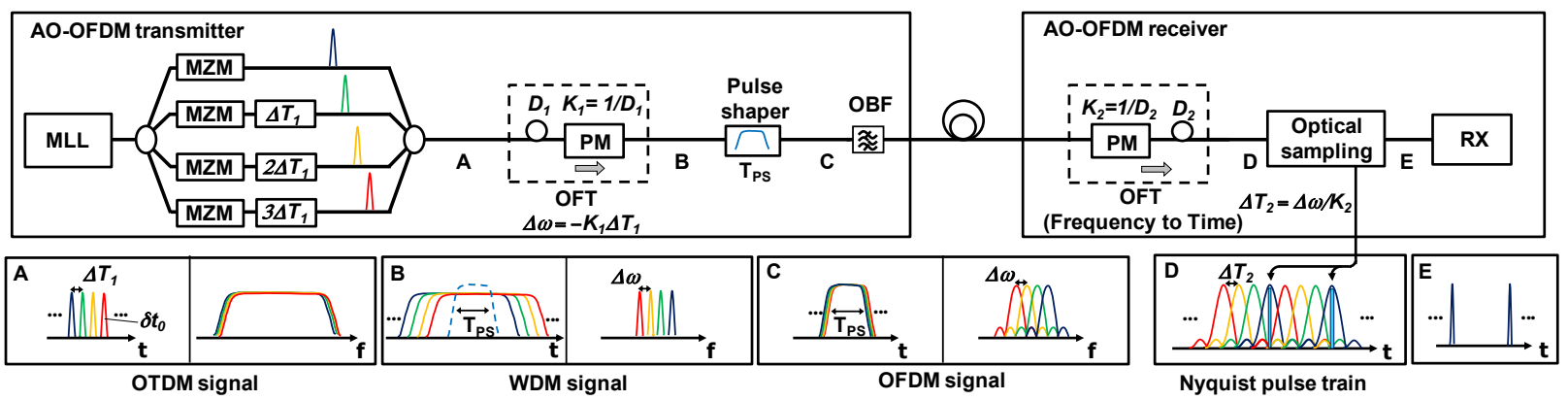

Fig. 1. The principle of all-optical OFDM transmission system using time-domain OFTs. 


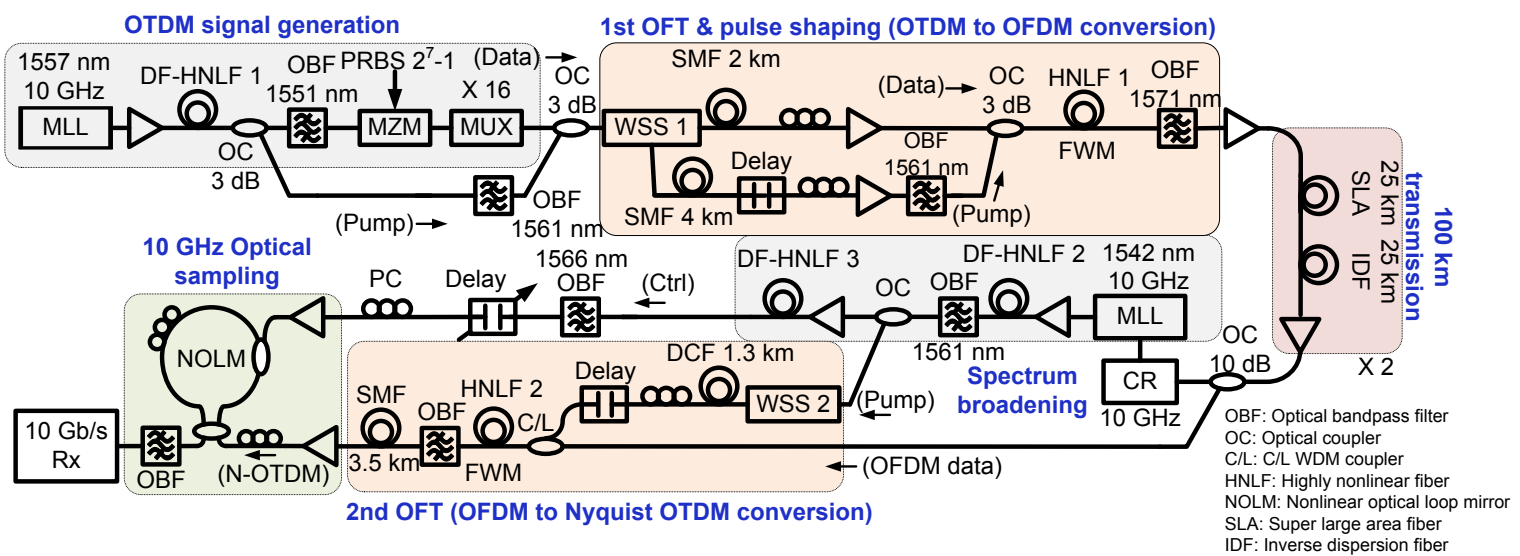

Fig. 2. Experimental setup for $160 \mathrm{Gbaud}$ all-optical OFDM transmission using time-domain OFT by four wave mixing (FWM).

dispersion medium $D_{l}$ (a K-D-K configuration). However, if the input pulse width $\delta t_{0}$ is very narrow such that $\left|\delta t_{0}{ }^{2} / 2 D_{I}\right|<<1$, then the first phase modulation stage can be omitted [8], which leads to the simpler $D-K$ configuration used here. After the OFT, the OTDM signal (A) in Fig. 1 is thus converted to a WDM signal with overlapped flattop waveforms (B) by complete OFT in the D-K time-lens system, and with channel spacing $\Delta \omega=-K_{l} \Delta T_{l}$. A rectangular pulse shaper (gate-width $T_{P S}$ ) is then used to select the center of the overlapped pulses, resulting in sincshaped OFDM subcarriers with spectral width given by $2 \pi / T_{P S}$, set equal to $\Delta \omega$ to achieve frequency orthogonality (C). Note that the complete OFT is only achieved within the finite time aperture $T_{P S}$ in the D-K configuration. Furthermore, a guard interval is introduced between each group of OTDM tributaries so that the pulse tails from neighboring bit slots within the gate-window are mapped to wavelengths well separated from the desired OFDM spectrum. These wavelengths are finally removed by an optical bandpass filter (OBF). In the receiver, a timedomain OFT is used to frequency-to-time convert the OFDM spectrum into a Nyquist-pulse OTDM (N-OTDM) signal with tributary spacing $\Delta T_{2}=\Delta \omega / K_{2}$. The N-OTDM signal is subsequently demultiplexed by sampling out each subcarrier at the inter-symbol-interference (ISI)-free point using a narrow optical sampling gate (D) [9]. Finally, the demultiplexed signal $(\mathbf{E})$ is detected by a base rate receiver. The proposed scheme allows simultaneous time-domain OFT processing of all OFDM subcarriers both in the transmitter and the receiver. Compared to other OFT/OIFT schemes for AO-OFDM [3-5], the system complexity does not increase with the number of subcarriers.

\section{Experimental setup and results}

The experimental setup is shown in Fig. 2. A $160 \mathrm{Gbit} / \mathrm{s}$ OTDM-DPSK signal with $1.7 \mathrm{ps}$ wide pulses, centered at $1551 \mathrm{~nm}$ with a guard interval of $44 \mathrm{ps}$ between each group of 16 tributaries is generated and further rectangular filtered with a wavelength selective switch (WSS1). The generated OTDM data signal then undergoes complete OFT in a D-K time lens. As the first step of the OFT process, the $160 \mathrm{Gbit} / \mathrm{s}$ OTDM signal is dispersed in a $2 \mathrm{~km}$ SSMF, resulting in overlapping 160 ps flat-top linearly-chirped pulses. The quadratic phase modulation and pulse shaping are here simultaneously achieved in a FWM process (HNLF 1) using 80 ps linearly chirped rectangular pump pulses $(1561 \mathrm{~nm}$ ), obtained through filtering in WSS1 and $4 \mathrm{~km}$ SSMF propagation. Fig. 3 (a) shows the spectrum after FWM. The center part of the idler is the 16 subcarrier OFDM spectrum with channel spacing 12.5 GHz, also shown in Fig. 3 (b). The empty bands in the idler spectrum in Fig. 3 (a) originate from the 44 ps guard-intervals in the OTDM signal. Fig. 3 (b) also shows the spectra obtained for individual OFDM subcarriers,
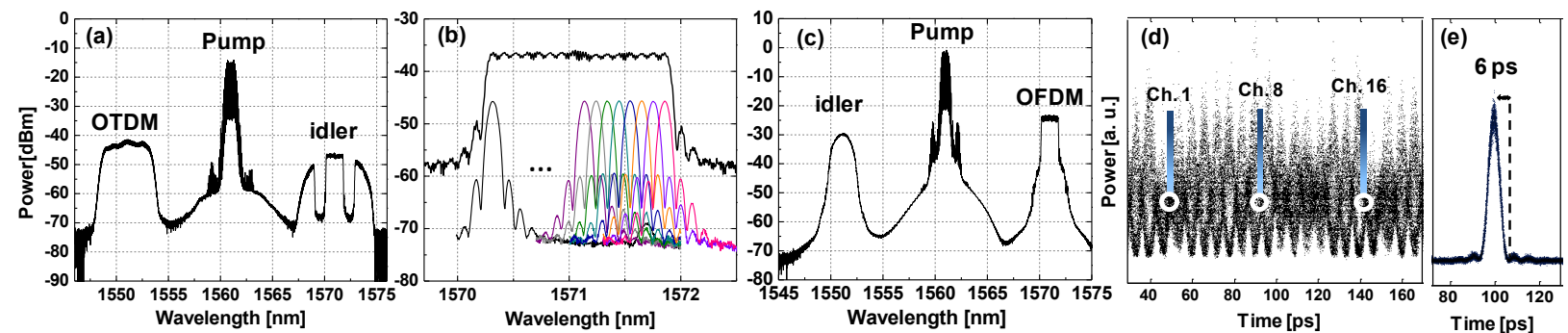

Fig. 3. Results of OTDM to OFDM conversion (a) spectrum after first FWM, (b) obtained OFDM spectrum and individual sinc-shaped subcarriers for different input OTDM tributaries. Results of OFDM to OTDM conversion (c) spectrum after second FWM, (d) obtained NOTDM signal, (e) single Nyquist tributary waveform. 

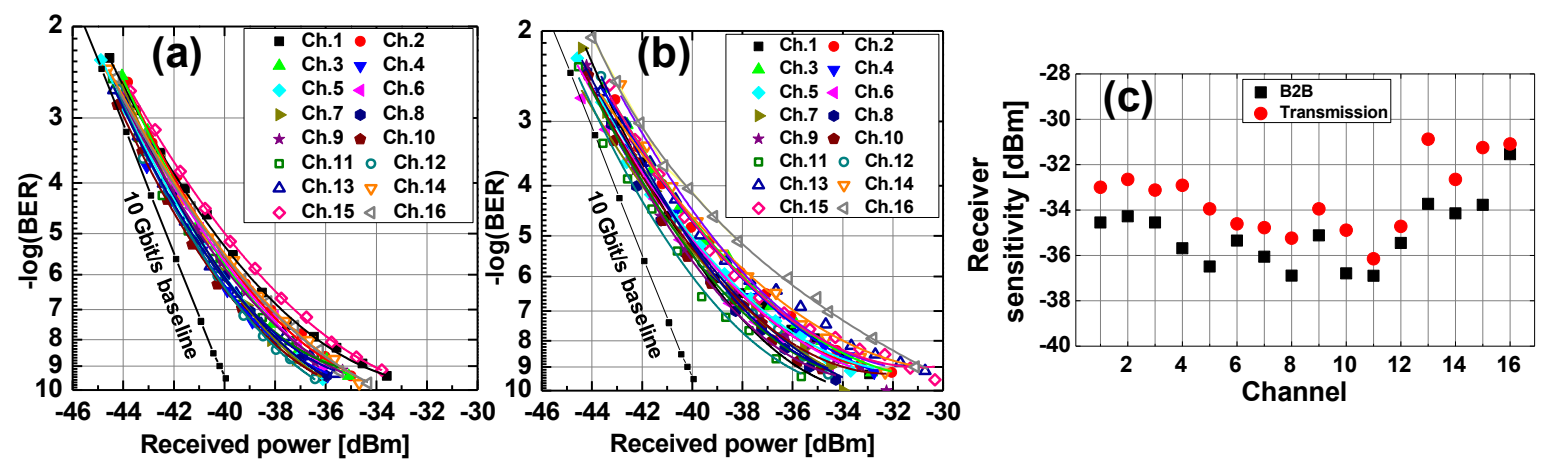

Fig. 4. BER measurements (10 Gbit/s DPSK) of 16 OFDM subcarriers (a) Reference measurement (b) $100 \mathrm{~km}$ transmission. (c) Receiver power sensitivities at $\mathrm{BER}=10^{-9} \mathrm{~B} 2 \mathrm{~B}$ and after $100 \mathrm{~km}$

where the characteristic sinc-shape can be observed. The 16-carrier OFDM spectrum is extracted by a $2.8 \mathrm{~nm}$ OBF $(1571 \mathrm{~nm})$ and launched into a $2 \times 50 \mathrm{~km}$ SLA-IDF dispersion-managed link. It can be noted that, as the $160 \mathrm{Gbit} / \mathrm{s}$ OTDM-DPSK signal is generated from a $10 \mathrm{Gbit} / \mathrm{s}$ PRBS $\left(2^{7}-1\right)$ signal and a PRBS $\left(2^{7}-1\right)$ maintaining passive fibredelay multiplexer, all 16 subcarriers in an OFDM symbol interval carry different information, resulting in decorrelated OFDM subcarriers, unlike the even and odd channel scheme [1-2].

In the receiver, a FWM-based OFT time lens (HNLF 2) is used for the OFDM to N-OTDM conversion, and a NOLM is employed for the $10 \mathrm{GHz}$ optical sampling using $1.1 \mathrm{ps}$ wide control pulses [9]. The FWM pump pulses $(1561 \mathrm{~nm})$ and NOLM control-pulses $(1566 \mathrm{~nm})$ are obtained via spectral broadening in DF-HNLFs and OBF filtering. The rectangular $90 \mathrm{ps}$ wide linearly-chirped pump pulses are obtained by filtering in WSS2 and $1.3 \mathrm{~km}$ DCF propagation. The spectrum after FWM is shown in Fig. 3 (c). The idler signal at $1551 \mathrm{~nm}$ is extracted by an OBF and propagated through $3.6 \mathrm{~km}$ SSMF, resulting in the 160 Gbaud N-OTDM signal shown in Fig. 3 (d). The tributary spacing is $6 \mathrm{ps}$, and the sinc-shaped waveform of a single tributary can be observed in Fig. 3 (e). The ISIfree point obtained in each tributary time-slot is sampled out using the NOLM, and the BER of each tributary (subcarrier) is measured in a $10-\mathrm{Gbit} / \mathrm{s}$ pre-amplified DPSK receiver.

The BER performance is shown in Fig. 4 . Fig. 4 (a) pertains to a reference system where the pump and control signals for the receiver-OFT are derived directly from the output of DF-HNLF1. A BER $<10^{-9}$ is obtained for all 16 subcarriers, confirming the proposed principle. The average power penalty for all 16 subcarriers at $B E R=10^{-9}$ is $4.2 \mathrm{~dB}$ compared to the $10 \mathrm{Gbit} / \mathrm{s}$ DPSK 50\%-RZ baseline. Fig. 4 (b) pertains to the full $100 \mathrm{~km}$ transmission system, including clock recovery $(\mathrm{CR})$ and pump/control pulse generation at the receiver. The $100 \mathrm{~km}$ transmission is also successful with BER $<10^{-9}$ for all 16 subcarriers. Fig. 4 (c) shows the receiver sensitivities of all channels at $\mathrm{BER}=10^{-9}$ for the $100-\mathrm{km}$ transmission (full system with $\mathrm{CR}$ ), and for the back-to-back (B2B) system (full system without $\mathrm{CR}$ and transmission). There is an average $1.6 \mathrm{~dB}$ transmission penalty, which is mainly attributed to additional jitter in the clock recovery from the OFDM signal.

\section{Conclusion}

We have proposed a novel simple all-optical OFDM transmission system based on time-domain OFT using optical time lenses for the OFDM subcarrier multiplexing and demultiplexing. We successfully demonstrated a real-time error-free $160 \mathrm{Gbit} / \mathrm{s}$ OFDM-DPSK system with 16 decorrelated data subcarriers, including a 100-km transmission. A $0.8 \mathrm{symbol} / \mathrm{s} / \mathrm{Hz}$ spectral efficiency was achieved, which is close to the single channel theoretical limit 1 symbol $/ \mathrm{s} / \mathrm{Hz}$.

Acknowledgements: OFS Denmark Aps, Danish Research Council FTP project TOR (ref.no. 12-127224).

\section{References}

[1] A. Sano et al., J. Lightwave Technol. 27, 3705-3713, 2009.

[2] D. Hillerkuss et al., Nature Photonics 5(6), 364-371, 2011.

[3] L. Du et al., Proc. OFC2013, PDP5B.9, 2013.

[4] D. Hillerkuss et al., Opt. Express, 18(9), 9324-9340, 2010.

[5] A. J. Lowery et al., Opt. Express 19(17), 15696-15704, 2011.

[6] M. Nakazawa et al., IEEE PTL. 16, 1059-1061, 2004.

[7] H. C. H. Mulvad et al. Proc. ECOC 2011,Mo.1.A.5, 2011

[8] E. Palushani., IEEE J. Quantum Electron, 45(11), 1317-1324, 2009.

[9] M. Nakazawa et al., Opt. Express, 20(2), 1129-1140, 2012. 